\title{
Endocrine Pathology
}

Editor

JUSTINE A. BARLETTA

\section{SURGICAL PATHOLOGY CLINICS}

www.surgpath.theclinics.com

Consulting Editor

JASON L. HORNICK

December 2019 • Volume 12 • Number 4 